anti-HTLV-1 antibody positive and negative cases.

\section{Discussion}

HHV-6 is a new member of the herpes virus group. ${ }^{1}$ The predominant affinity of CD4 positive $T$ cells ${ }^{4}$ and a high incidence of latent infection with the virus suggest that $\mathrm{HHV}-6$, like other such viruses, may cause specific lymphocyte related disease or may have some role in opportunistic infection. Recently, Josephs et al and Jarrett et al reported that HHV-6 DNA had been detected in three of 82 B cell lymphomas ${ }^{8}$ and two of 117 lymphomas using Southern blot analysis.? Buchbinder et al and Torelli et al also reported detecting HHV-6 DNA in 23 of 25 different malignant lymphomas, ${ }^{9}$ three of 25 Hodgkin's lymphomas, and none of 41 nonHodgkin's lymphomas ${ }^{6}$ using PCR methods. A definite association between malignant lymphoma and HHV-6 however, was still unclear.

In our study we found amplified HHV-6 DNA using PCR in $50 \cdot 0-68.8 \%$ of samples studied, but found no clear differentiation in rates of detection among different types of malignant lymphoma. Using Southern blot analysis, we were unable to detect any HHV6 DNA. Using PCR, Kondo et al identified HHV-6 infected monocyte/macrophage cells in lymphadenopathies. ${ }^{10} \mathrm{We}$ also clarified that HHV-6 infected CD68-positive monocyte/macrophage cells existed in a latent form, using double staining in situ hybridisation and immunohistochemistry methods. ${ }^{11}$ We therefore deduced that the presence of
HHV-6 DNA shown by PCR in lymphoid malignancies was derived from latent infection. In this study we were unable to determine the HHV- 6 positive cell subsets, but the HHV-6 positive cells might be infected in monocyte/macrophage cells as they are the same as in benign lymphadenitis.

This work was supported by Grant 1992 from Fukuoka University Hospital Clinical Research Fund.

1 Salahuddin S, Ablashi DV, Markham PD, et al. Isolation of new virus, HTLV, in patients with lymphoproliferaof new virus, HTLV, in patients with lymp

2 Lusso P, Salahuddin SZ, Ablashi DV, et al. Diverse tropism of human B-lymphotropic virus (human herpesvirus-6). Lancet 1987;ii:243-4.

3 Yamanishi K, Toshiomi O, Kimiyasu S, et al. Identification of human herpesvirus- 6 as a causal agent for exanthema subitum. Lancet 1988;i:1065-7.

4 Takahashi K, Sonoda S, Higashi K, et al. Predominant CD4 T-lymphocyte tropism of human herpesvirus-6 related virus. $1989 ; 63: 3161-3$

5 Jarrett RF, Gledhill S, Qureshi F, et al. Identification of human herpesvirus 6-specific DNA sequences in two patients with non-Hodgkin's lymphoma. Leukemia patients with non.

6 Torelli G, Marasca R, Luppi M, et al. Human herpesvirus-6 in human lymphomas: identification of specific sequences in Hodgkin's lymphomas by polymerase chain reaction. Blood 1991;77:2251-8.

7 Borisch B, Ellinger K, Neipel F, et al. Lymphadenitis and lymphoproliferative lesions associated with human herpesvirus-6 (HHV-6). Virchows Arch (Cell Pathol) 1991;61:179-87.

8 Josephs SF, Buchbinder A, Streicher HZ, et al. Detection of human B-lymphotropic virus (human herpesvirus-6) sequences in B cell lymphoma tissue of three patients. Leukemia 1988;2:132-5.

9 Buchbinder A, Josephs SF, Ablashi DV, et al. Polymerase chain reaction amplification and in situ hybridization for chain reaction amplification and in situ hybridization for the detection of Human

10 Kondo $\mathrm{K}$, Kondo T, Okuno $\mathrm{T}$, et al. Latent human herpesvirus-6 infection of human monocytes/macrophages. $\ngtr$ Gen Virol 1991;72:1401-8.

11 Sumiyoshi Y, Kikuchi M, Ohshima K, et al. Human Herpesvirus-6 genomes in histiocytic necrotizing lymphadenitis (Kikuchi's disease) and other forms of lymphadenitis. Am $\mathcal{F}$ Clin Pathol 1993;99:609-14.

\title{
Inaccurate haemoglobin estimation in Waldenström's macroglobulinaemia: unusual reaction with monomeric IgM paraprotein
}

\author{
M J Goodrick, R J Boon, R J D Bishop, J A Copplestone, A G Prentice
}

\footnotetext{
Department of Haematology Derriford Hospital Plymouth

$M$ J Goodrick R J Boon R J D Bishop J A Copplestone A G Prentice

Correspondence to: Dr M J Goodrick, South Western Regional Transfusion Centre, Southmead Road, Bristol BS10 5ND

Accepted for publication 5 July 1993
}

\begin{abstract}
Automated blood counts from a patient with Waldenström's macroglobulinaemia repeatedly failed critical limit standards set for mean cell haemoglobin concentration and mean cell haemoglobin. Haemoglobin estimation was higher than that suggested by clinical examination, symptoms, and the spun haematocrit. This was found to be due to an interaction between the Coulter lysing agent and monomeric IgM paraprotein in the
\end{abstract}

patient's plasma, creating a precipitate which was optically dense at $525 \mathrm{~nm}$.

(F Clin Pathol 1993;46:1138-1139)

Automated blood analyses have greatly increased the availability and accuracy of full blood counts. Haemoglobin measurements are made by converting haemoglobin to cyanmethaemoglobin and then measuring absorbance at $525 \mathrm{~nm}$. The mean cell 
Estimation of $O D$ at $525 \mathrm{~nm}$ of IgM and IgG fractions by Quick-Sep separation with and without addition of Lyse-S in test and control plasmas

\begin{tabular}{llll}
\hline & & \multicolumn{2}{l}{ OD at $525 \mathrm{~nm}$} \\
Plasma fraction & Sample & - Lyse-S & + Lyse-S \\
\hline IgG & Patient & 0.072 & 2.250 \\
& IgG myeloma & 0.056 & 0.060 \\
& Waldenström's macroglobulinaemia & 0.083 & 0.223 \\
IgM & control & 0.062 & 0.067 \\
& Patient & 0.074 & 0.082 \\
& IgG myeloma & 0.106 \\
& Waldenström's macroglobulinaemia & 0.064 & \\
\hline
\end{tabular}

haemoglobin $(\mathrm{MCH})$ and mean cell haemoglobin concentration (MCHC) are derived from the directly measured haemoglobin, red cell count, and mean cell volume. ${ }^{1}$ Other factors which alter the optical density of a blood sample, such as lipaemia or high white cell counts, are well recognised to confound haemoglobin estimations using this technique. ${ }^{1}$

It was noted in our laboratory that samples from a patient with an IgM secreting low grade non-Hodgkin's lymphoma (Waldenström's macroglobulinaemia) repeatedly failed critical limits for both MCHC and MCH using Technicon H6000, Coulter JS, and Coulter STKR automated cell counters.

Clinical symptoms and manual haematocrits, performed by a spin technique, indicated that the patient was more anaemic than was suggested by the reported haemoglobin concentration. If the plasma from the sample was removed and replaced by an equal volume of Isoton, the measured haemoglobin was $40 \mathrm{~g} / 1$ lower than first estimated. Treatment of the plasma with 2-mercaptoethanol (2ME) did not correct this discrepancy. No cryoglobulin could be detected.

\section{Method and Results}

The patient's plasma was separated from fresh EDTA samples, mixed with Coulter lysing solution (Lyse-S), and analysed through a Coulter STKR. The plasma treated in this way gave a "haemoglobin" estimation of $40 \mathrm{~g} / 1$, confirming that a plasma fraction was interacting with the lysing reagent to cause the false reading. The patient's plasma was then separated into IgG and IgM fractions using Quick-Sep columns, according to the manufacturer's protocol (Isolab Inc, Drawer 4350, Akron, Ohio, USA). As controls, plasma was obtained from a known IgG myeloma and from another patient with Waldenström's macroglobulinaemia in whom haemoglobin estimates were felt to be accurate and these were separated in the same way. The optical density (OD) of each fraction was then measured at $525 \mathrm{~nm}$ before and after the addition of Lyse-S at a 1 in 7 dilution. A sharp increase in OD of the test IgG fraction was seen. There was also a small increase in the OD of the IgG fraction from plasma from the control with Waldenström's macroglobulinaemia but no change in the IgG myeloma control (table).

A precipitate formed when the patient's IgG fraction was incubated at $4^{\circ} \mathrm{C}$. The precipitate was separated from the supernatant fluid by centrifugation, washed twice in $\mathrm{pH}$ 8.6 buffer, and resuspended. The supernatant fluid, the resuspended precipitate, and the Ig fractions from Quick-Sep separation were electrophoresed on agarose at $\mathrm{pH} 8.6$, and immunofixation, using anti-IgG and anti IgM, was performed. This showed that IgM could be detected in both the IgG and IgM fractions of the patient's plasma and in the precipitate. IgG was present in the plasma IgG fraction and in the supernatant fluid, but none could be detected in the precipitate.

\section{Conclusion}

It is concluded that there is a reaction between Lyse-S and the paraprotein which passed with the IgG fraction in Quick-Sep filtration. This results in an increase in plasma $\mathrm{OD}$ and consequently in inaccurate haemoglobin estimation. In view of its mobility with the IgG fraction and the lack of removal with $2 \mathrm{ME}$, this is likely to represent monomeric IgM.

Patients with Waldenström's macroglobulinaemia are known to produce nonpentameric IgM components in varying amounts, ${ }^{23}$ probably because of defective polymerisation. ${ }^{4}$ No similar reactions have been described before, but the findings of small increases in the OD of the plasma fractions in the patient with Waldenström's macroglobulinaemia selected as a control suggests that the difference may be one of degree; the more monomeric IgM formed, the greater the chance of detecting similar interactions. This would merit further assessment.

In the case described, in order to obtain an accurate haemoglobin measurement for clinical use, all the plasma has to be removed from a spun EDTA sample. This is replaced with an equal volume of Isoton and the manipulated sample is then processed through the Coulter STKR in the normal manner.

1 Hall R, Malia RG. Automated blood cell analysis. In: Medical laboratory haematology. London: Butterworths, 1984:192-5.

2 Soloman A, McLaughlin CL. Biosynthesis of low molecular weight (7S) and high molecular weight (19S) iman we

3 Davis AL, Shulman MJ. IgM-Molecular requirements for its assembly and function. Immunol Today 1989; 10:118-28.

$4 \mathrm{Xu} \mathrm{H}$, Roberts-Thomson PJ. Circulating low molecular weight IgM-a disease marker in autoimmune, infective, immunodeficient and B-cell lymphoproliferative disorders. Disease Markers 1992;10:115-41. 